PSYCHOMETRIKA-VOL. 30, NO. 4

DECEMBER, 1965

\title{
A SET OF INEQUALITIES IN FACTOR ANALYSIS
}

\author{
J. N. Darroch \\ UNIVERSITY OF MICHIGAN
}

Inequalities relating the communalities to the multiple-correlation coefficients are derived. They are stronger than the well-known inequalities which have played an important role in factor analysis for the past thirty years. Necessary and sufficient conditions for equality are obtained.

\section{Introduction}

Let $\mathbf{\Sigma}=\left[\sigma_{i i}\right]$ denote the correlation matrix of $\mathrm{x}=\left[\begin{array}{llll}x_{1} & x_{2} & \cdots & x_{p}\end{array}\right]^{\prime}$. We shall suppose that $\Sigma$ is nonsingular and therefore positive definite. (If, to the contrary, $\mathbf{\Sigma}$ is of rank $r(<p)$ then there are only $r$ linearly independent variables $x_{1}, x_{2}, \cdots, x_{r}$, say, and the others are redundant.)

Next let

$$
\mathbf{\Sigma}=\boldsymbol{\Gamma}+\mathbf{\Delta}
$$

where

$$
\Delta=\left[\begin{array}{cccc}
\delta_{1}^{2} & 0 & \cdots & 0 \\
0 & \delta_{2}^{2} & \cdots & 0 \\
\vdots & \vdots & & \vdots \\
0 & 0 & \cdots & \delta_{p}^{2}
\end{array}\right] \quad 0 \leq \delta_{i}^{2} \leq 1, \quad 1 \leq i \leq p
$$

and $\boldsymbol{\Gamma}=\mathbf{\Sigma}-\boldsymbol{\Delta}$ is positive semi-definite. The factor-analysis interpretation of (1) is that

$$
x=y+z,
$$

where $\mathbf{y}=\left[\begin{array}{llll}y_{1} & y_{2} & \cdots & y_{p}\end{array}\right]^{\prime}$ has covariance matrix $\boldsymbol{\Gamma}$ and is uncorrelated with $\mathrm{z}=\left[\begin{array}{llll}z_{1} & z_{2} & \cdots & z_{p}\end{array}\right]^{\prime}$ which has covariance matrix $\Delta$, so that $z_{i}$ is uncorrelated with $z_{j}, 1 \leq i \leq j \leq p$. The variable $y_{i}$ is called the common-factor component of $x_{i}$ and $\operatorname{var}\left(\overline{y_{i}}\right)=1-\delta_{i}^{2}$ is the communality of $x_{i}$ while $z_{i}$ is the specific factor of $x_{i}$ and $\operatorname{var}\left(z_{i}\right)=\delta_{i}^{2}$ is the uniqueness of $x_{i}$.

Let $\rho_{i}$ denote the multiple-correlation coefficient of $x_{i}$ with the remaining $p-1$ variables. Then

$$
\rho_{i}<1
$$$$
1 \leq i \leq p \text {, }
$$ 
because $\boldsymbol{\Gamma}$ is nonsingular. Roff [3] pointed out and Dwyer [1] proved that (3)

$$
1-\delta_{i}^{2} \geq \rho_{i}^{2}
$$$$
1 \leq i \leq p \text {. }
$$

In this paper we derive a set of stronger inequalities than (3).

\section{The Inequalities}

Write

$$
\mathbf{\Sigma}=\left[\begin{array}{cc}
1 & \boldsymbol{\sigma}_{1}^{\prime} \\
\boldsymbol{\sigma}_{1} & \mathbf{\Sigma}_{11}
\end{array}\right],
$$

where $\boldsymbol{\Gamma}_{11}$ is the covariance matrix of $\mathbf{x}_{1}=\left[\begin{array}{llll}x_{2} & x_{3} & \cdots & x_{p}\end{array}\right]^{\prime}$ and define

$$
\beta_{1}=\Sigma_{11}^{-1} \delta_{1} \text {. }
$$

Thus $\beta_{1}=\left[\begin{array}{llll}\beta_{12} & \beta_{13} & \cdots & \beta_{1 p}\end{array}\right]^{\prime}$ is the vector of regression coefficients of $x_{1}$ on $\mathbf{x}_{1}$. Now

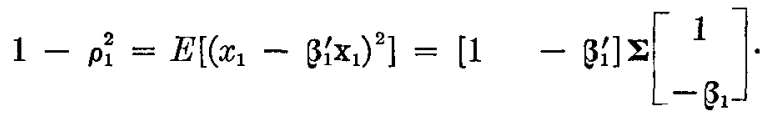

Therefore

$$
1-\rho_{1}^{2}=\left[\begin{array}{ll}
1 & -\beta_{1}^{\prime}
\end{array}\right] \Gamma\left[\begin{array}{c}
1 \\
-\beta_{1}
\end{array}\right]+\left[\begin{array}{ll}
1 & -\beta_{1}^{\prime}
\end{array}\right] \Delta\left[\begin{array}{c}
1 \\
-\beta_{1}
\end{array}\right] .
$$

The second term on the right of (4) is $\delta_{1}^{2}+\beta_{12}^{2} \delta_{2}^{2}+\beta_{13}^{2} \delta_{3}^{2}+\cdots+\beta_{1 p}^{2} \delta_{p}^{2}$ and, since $\boldsymbol{\Gamma}$ is positive semi-definite, the first term is nonnegative. Applying the same argument to $1-\rho_{2}^{2}, \cdots, 1-\rho_{p}^{2}$, we have the following set of $p$ inequalities

$$
1-\rho_{i}^{2} \geq \delta_{i}^{2}+\sum \beta_{i j}^{2} \delta_{i}^{2} \quad 1 \leq i \leq p,
$$

where $\beta_{i}$ is the coefficient of $x_{j}$ in the regression of $x_{i}$ on the remaining $p-1$ variables.

\section{Conditions for Equality}

Suppose that

$$
1-\rho_{1}^{2}=\delta_{1}^{2}+\sum_{i>1} \beta_{1 i}^{2} \delta_{i}^{2} .
$$

Then $\boldsymbol{\alpha}_{1}^{\prime} \boldsymbol{\Gamma} \boldsymbol{\alpha}_{1}=0$, where $\boldsymbol{\alpha}_{1}^{\prime}=\left[1-\beta_{1}^{\prime}\right]$. But, since $\boldsymbol{\Gamma}$ is positive semi-definite, $\boldsymbol{\alpha}_{1}^{\prime} \boldsymbol{\Gamma} \boldsymbol{\alpha}_{1}=0$ only if $\boldsymbol{\Gamma} \boldsymbol{\alpha}_{1}=0$. Therefore (6) holds if and only if

$$
\left[\begin{array}{cccc}
1-\delta_{1}^{2} & \boldsymbol{\sigma}_{1}^{\prime} & \\
\boldsymbol{\delta}_{1} & \mathbf{\Sigma}_{11}- & \mathbf{\Delta}_{1}
\end{array}\right]\left[\begin{array}{c}
1 \\
-\beta_{1}
\end{array}\right]=\left[\begin{array}{l}
0 \\
0
\end{array}\right],
$$


where

Equations (7) are

$$
\boldsymbol{\Delta}_{1}=\left[\begin{array}{cccc}
\delta_{2}^{2} & 0 & \cdots & 0 \\
0 & \delta_{3}^{2} & \cdots & 0 \\
\vdots & \vdots & & \vdots \\
0 & 0 & \cdots & \delta_{p}^{2}
\end{array}\right]
$$

$$
\begin{array}{r}
1-\delta_{1}^{2}-\boldsymbol{d}_{1}^{\prime} \boldsymbol{\beta}_{1}=0 \\
\boldsymbol{d}_{1}-\mathbf{\Sigma}_{11} \boldsymbol{\beta}_{1}+\Delta_{1} \boldsymbol{\beta}_{1}=0
\end{array}
$$

However

$$
\begin{aligned}
\sigma_{1}^{\prime} \beta_{1}=\sigma_{1}^{\prime} \Sigma_{11}^{-1} \sigma_{1} & =\rho_{1}^{2} \\
\boldsymbol{\sigma}_{1} & =\mathbf{\Sigma}_{11} \beta_{1} .
\end{aligned}
$$

Therefore (6) holds if and only if

$$
\delta_{1}^{2}=1-\rho_{1}^{2}
$$

and

$$
\beta_{12} \delta_{2}^{2}=\beta_{13} \delta_{3}^{2}=\cdots=\beta_{1 p} \delta_{p}^{2}=0 .
$$

Equations (9) state that, for each $j, 2 \leq j \leq p$, either $\delta_{j}^{2}=0$ or $\beta_{1 j}=0$.

At this stage it is worth noting the connection between the regression coefficients $\beta_{i j}$ and the matrix $\mathbf{\Sigma}^{-1}$. Writing

$$
\mathbf{\Sigma}=\left[\begin{array}{ll}
1 & \boldsymbol{\sigma}_{1}^{\prime} \\
\mathbf{d}_{1} & \mathbf{\Sigma}_{11}
\end{array}\right],
$$

we have

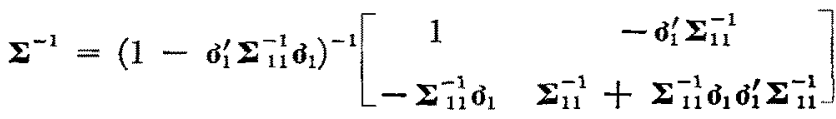

$$
\begin{aligned}
& =\left(1-\rho_{1}^{2}\right)^{-1}\left[\begin{array}{cc}
1 & -\beta_{1}^{\prime} \\
-\beta_{1} & \Sigma_{11}^{-1}+\beta_{1} \beta_{1}^{\prime}
\end{array}\right] \text {. }
\end{aligned}
$$

From the form of the first row of $\mathbf{\Sigma}^{-1}$ it follows that

(10)

$$
\mathbf{\Sigma}^{-1}=\mathbf{D}^{-1}\left[\begin{array}{ccccc}
1 & -\beta_{12} & -\beta_{13} & \cdots & -\beta_{1 p} \\
-\beta_{21} & 1 & -\beta_{23} & \cdots & -\beta_{2 p} \\
-\beta_{21} & -\beta_{32} & 1 & \cdots & -\beta_{3 p} \\
\vdots & \vdots & \vdots & & \vdots \\
-\beta_{p 1} & -\beta_{p 2} & -\beta_{p 3} & \cdots & 1
\end{array}\right]=\mathbf{D}^{-1}\left[\begin{array}{c}
\boldsymbol{\alpha}_{1}^{\prime} \\
\boldsymbol{\alpha}_{2}^{\prime} \\
\boldsymbol{\alpha}_{3}^{\prime} \\
\vdots \\
\alpha_{p}^{\prime}
\end{array}\right], \text { say, }
$$


where $\mathbf{D}$ in the diagonal matrix whose $i$ th diagonal element is $1-\rho_{i}^{2}$. Thus $\beta_{i j}=0, i \neq j$, if and only if the $(i, j)$ element of $\mathbf{\Sigma}^{-1}$ is zero.

We see from (8) that there is equality in any member of (5) if and only if there is equality in the corresponding member of (3).

\section{Conditions for $p-m$ Equalities}

In this section we shall obtain necessary and sufficient conditions for equality in the last $p-m$ inequalities of (5) (and therefore of (3)). Guttman [2] obtained sufficient conditions for equality in the last $p-m$ inequalities. of (3) (and therefore of (5)). The end results of this section are closely related. to Guttman's but the method of analysis is different.

Let

$$
S=\{1,2, \cdots, m\}, \quad T=\{m+1, m+2, \cdots, p\} .
$$

Then we know that

$$
1-\rho_{i}^{2}=\delta_{i}^{2}+\sum_{j \neq i} \beta_{i j}^{2} \delta_{i}^{2} \quad i \varepsilon T
$$

if and only if

$$
\delta_{i}^{2}=1-\rho_{i}^{2} \quad i_{\varepsilon} T
$$

and

$$
\sum_{j \in S} \beta_{i j}^{2} \delta_{j}^{2}+\sum_{i \varepsilon T, j \otimes i} \beta_{i j}^{2} \delta_{j}^{2}=0, \quad i \varepsilon T .
$$

Equation (13), taken in conjunction with (12), holds if and only if

$$
\beta_{i j} \delta_{i}=0 \quad i \varepsilon T, \quad j \varepsilon S,
$$

and

$$
\beta_{i i}=0 \quad i \varepsilon T, \quad j \varepsilon T, \quad i \neq j .
$$

Condition (15) states that the $(i, j)$ element of $\mathbf{\Sigma}^{-1}$ is zero for all $i \varepsilon T, j \varepsilon T$, $i \neq j$. One way of describing this is to say that, given $x_{1}, x_{2}, \cdots, x_{m}$, the variables $x_{m+1}, x_{m+2}, \cdots, x_{p}$ are uncorrelated (partially). This is clearly a very special situation. (In particular, there is equality in all $p$ inequalities if and only if $x_{1}, x_{2}, \cdots, x_{p}$ are completely uncorrelated, that is $\mathbf{\Sigma}=\mathbf{I}$.) When (12), (14), and (15) hold, $\boldsymbol{\Gamma}=\mathbf{\Sigma}-\boldsymbol{\Delta}$ is at most of rank $m$ for

$$
\boldsymbol{\Gamma} \boldsymbol{\alpha}_{i}=0
$$

and, from (10), the vectors $\boldsymbol{\alpha}_{m+1}, \cdots, \boldsymbol{\alpha}_{p}$ are linearly independent.

So far we have pointed out that (12), (14), and (15) are necessary and sufficient for (11). Now (14) holds in particular if

$$
\delta_{j}=0 \quad j \varepsilon S .
$$


When (12), (15), and (16) are satisfied $\boldsymbol{\Gamma}$ is exactly of rank $m$ for, using an obvious notation, we have

$$
\mathbf{\Sigma}=\left[\begin{array}{ll}
\mathbf{\Sigma}_{S S} & \mathbf{\Sigma}_{S T} \\
\mathbf{\Sigma}_{T S} & \mathbf{\Sigma}_{T T}
\end{array}\right]=\left[\begin{array}{cc}
\mathbf{\Sigma}_{S S} & \mathbf{\Sigma}_{S T} \\
\mathbf{\Sigma}_{T S} & 0
\end{array}\right] .
$$

\section{Discussion}

Guttman [2] proved an important limiting relationship between the communalities $1-\delta_{i}^{2}$ and the multiple-correlation coefficients $\rho$. Namely that, if $q$ is the rank of $\Gamma$ and $q / p \rightarrow 0$ as $p \rightarrow \infty$, then $\left(1-\delta_{i}^{2}\right) / \rho_{i}^{2} \rightarrow 1, i=$ $1,2, \cdots, p$. Thus the communality $1-\delta_{i}^{2}$ may be characterised as the squared multiple-correlation coefficient of $x_{i}$ with an infinite set of "relevant" variables. This property, and the fact that in very special situations it is possible for $1-\delta_{i}^{2}$ to equal $p_{i}^{2}$ for some values of $i$, led Guttman to call $\rho_{i}^{2}$ the "best possible" systematic estimate of $1-\delta_{i}^{2}$ in the practical case of a finite number of variables. While it is usually realized that the use of these estimates is strictly illegitimate in the sense that they lead to a $\mathbf{\Gamma}$ which is nonnegative definite and therefore cannot be a covariance matrix, the extent to which they are illegitimate may now be better judged from the amount by which they contradict the $p$ inequalities (5).

In this paper we have only been concerned with helping to demarcate the region of legitimate communalities and not with any criteria which propose a particular point in this region as the communality solution. We hope to treat this aspect in a later paper.

\section{REFERENCES}

[1] Dwyer, P. S., The contribution of an orthogonal multiple factor solution to multiple correlation. Psychometrika, 1939, 4, 163-171.

[2] Guttman, L. "Best possible" systematic estimates of communalities. Psychometrika, $1956,21,273-285$.

[3] Roff, M. Some properties of the communality in multiple factor theory. Psychometrika, 1935, 1, 1-6. 